\title{
Neural correlates of individual differences in aversion to risk and choice inconsistencies
}

\author{
Manon E. Jaquerod ${ }^{\mathrm{a}}$, Alessandra Lintas ${ }^{\mathrm{a}, \mathrm{b}}$, Gabriele Gratton ${ }^{\mathrm{c}, \mathrm{d}}$, Monica Fabiani ${ }^{\mathrm{c}, \mathrm{d}}$, \\ Kathy A. Low ${ }^{\mathrm{d}}$, Philippe N. Toblere,f ${ }^{\mathrm{e}}$, Alessandro E. P. Villa ${ }^{\mathrm{a}}$ \\ ${ }^{a}$ Neuroheuristic Research Group, University of Lausanne, 1015 Lausanne, Switzerland \\ ${ }^{b}$ LABEX, Faculty of Business and Economics, University of Lausanne, 1015 Lausanne, \\ Switzerland \\ ${ }^{\mathrm{c}}$ Department of Psychology, University of Illinois at Urbana-Champaign, Champaign, IL, \\ 61820, USA \\ ${ }^{\mathrm{d} B e c k m a n}$ Institute for Advanced Science and Technology, University of Illinois at Urbana- \\ Champaign, Urbana, IL 61801, USA \\ ' Zurich Center for Neuroeconomics, Department of Economics, University of Zurich, 8006 \\ Zurich, Switzerland \\ ${ }_{\mathrm{f}}$ Neuroscience Center Zurich, University of Zurich, Swiss Federal Institute of Technology \\ Zurich, Zurich, Switzerland
}

\section{Adress correspondence to:}

Manon E. Jaquerod

University of Lausanne

1015, Lausanne

Switzerland

Phone: +41.21 .692 .33 .00$

Email: manon.jaquerod@unil.ch

\section{Competing interest statement}

The authors declare that there are no conflicts of interest.

\section{Acknowledgements}

We acknowledge financial support by the Swiss National Science Foundation (SNSF), grant POLAP1_178329 to MEJ. PNT was supported by the SNSF (grants 10001C_188878, 100019_176016, and 100014_165884). GG, MF, and KAL were supported by grant RF1 AG062 $\overline{6} 66$ from the National Institute on Aging. 


\begin{abstract}
Most people tend to prefer smaller certain gains to large uncertain gains when making financial choices (risk aversion). However, attitudes toward risk vary greatly between individuals, and over time within individuals. Consistent behavior may reflect the adoption by the individual of a simple or automatized heuristic which reduces the subject's uncertainty about the outcome of a behavioral choice. In contrast inconsistent behavior may reflect the adoption of a "fuzzy" logic, likely leaving high levels of uncertainty in the participant making the choice. Therefore, inconsistent behavior may often be associated with greater risk aversion. The use of simple/automatized heuristics may also lead to increased reliance on fast brain processes, whereas fuzzy heuristic may lead to lingering uncertainty. These two modes of processing may therefore lead to different brain dynamics. To examine these dynamics we recorded event-related brain potentials (ERPs) from 22 adults participants engaged in a task requiring choices between certain (but often smaller) gains and an uncertain (but often bigger) gains. Behavioral analyses allowed us to quantify choice consistency and risk aversion for each individual. Choice consistency was related to the amplitude of P200; risk aversion was related to modulation of the medial frontal negativity (MFN) as a function of choice uncertainty, to the amplitude of a late positive potential (LPP). These findings are consistent with the idea that differences in individuals' behavior when making financial choices may reflect variations in the type of heuristics they adopt, which in turn may may be reflected in differences in brain dynamics.
\end{abstract}

Keywords: Decision making, ERPs, Risk aversion, inconsistency, MFN, neuroeconomics 


\section{Introduction}

Most individuals exhibit aversion to risks when possible financial outcomes are positive (risk aversion) but seek risks when dealing with potential losses (Kahneman \& Tversky, 1979). In other words, most individuals tend to prefer a small certain gain to a large but uncertain one, and a large but uncertain loss to a small certain one. This may reflect a strategy that has the final goal of minimizing the probability of a disappointing outcome and the stress associated with it. Notwithstanding such tendencies, a great diversity in behavior can be observed (Hey, Does repetition improve consistency?, 2001; Frey, Johnson, \& De Neys, 2018). When financial choices are made, behavior is the outcome of a subjective valuation process (Kable \& Glimcher, 2009) and can reflect one's hidden "preferences" in particular contexts. Most optimal decision-making theories assume that preferences rely on stable valuations of each alternative choice. However, subjective valuation is in fact a dynamic process (Louie, Khaw, \& Glimcher, Normalization is a general neural mechanism for context-dependent decision making, 2013). The concept of choice inconsistency (i.e., a choice that is not consistent with a person's stated preferences) has been introduced to address this gap. Inconsistencies are ubiquitous in contexts characterized by repeated decision-making choices (Grether \& Plott, 1979; Kachelmeier \& Shehata, 1992; Seidl, 2002; Tsetsos, Usher, \& Chater, 2010). To account for this behavior, investigators have proposed that the decision maker's choice process is stochastic in nature (Harless \& Camerer, 1994; Hey \& Orme, b, 1994), leading to the development of probabilistic or stochastic models of choice (Intriligator, 1973; Mattsson \& Weibull, 2002; McFadden, 2005; Rieskamp, 2008; Blavatskyy \& Pogrebna, 2010; Ryan, 2018).

Neuroscientific methods have provided insights about value-based decision-making processes and recent work indeed supports the hypothesis that choice valuation may be 
stochastic (Webb, Levy, Lazzaro, Rutledge, \& Glimcher, 2019; Kurtz-David, Persitz, Webb, \& Levy, 2019). Nevertheless, the degree of choice consistency may itself be variable across people, reflecting individual differences in the neurocognitive processes leading to each specific choice. For instance, some individuals may operate on the basis of a very precise mathematical algorithm, leading to a specific choice, whereas others may adopt a "fuzzy logic" based on relatively imprecise representations of the value of different options. The first group may show great consistency across choices, whereas the second may show less. Importantly, the adoption of one or the other of these approaches may give rise to greater or smaller uncertainty about the average outcome of the strategy, which may, in turn, determine the degree of emotional involvement and risk aversion while performing a financial choice task. The aim of this study is to describe individual differences in the two dimensions of risk aversion and choice consistency and investigate which aspects of the subjective valuation process are reflected by the observed behaviors and underlying neural responses.

The mathematical characterization of the subjective value assigned to an option is labeled "utility". Therefore, we use the term utilitarian stimulus to refer to a stimulus that provides a choice between two options, associated with differential outcome values. In the context of risky decision making, one option (the risky option) can have two possible outcomes with a given probability of occurrence (e.g., 50-50\%). Various features of contextual information such as expected value (EV) of the risky option (i.e., the value you expect to obtain, on average, with the risky option) and its intrinsic risk (i.e., defined here as the range between the two possible outcomes) impact the subjective valuation of utilitarian stimuli (Tobler, O'Doherty, Dolan, \& Schultz, 2007). Valuation is hypothesized to be an adaptive process (Louie, Khaw, \& Glimcher, Normalization is a general neural mechanism for context- 
dependent decision making, 2013). Adaptation mechanisms are thought to work by adjusting the sensitivity of neural circuits in value-processing regions (such as the striatum, the anterior insula and the ventromedial prefrontal cortex) (Louie, Glimcher, \& Webb, Adaptive neural coding: from biological to behavioral decision-making, 2015). Previous ERP research on decision making tasks has shown that the valuation of utilitarian stimuli, includes both early (100-200 ms after stimulus-onset) and late processes (450-650 ms after stimulus onset), which are thought to impact choice behavior (Harris, Hare, \& Rangel, 2013; Gu, Zhang, Luo, \& Broster, 2018).

Neuroimaging methods have provided insights about the brain structures whose activity may be relevant to risky decision making. Animal studies have shown the involvement of lateral prefrontal cortex (IPFC) and parietal cortex in choice selection and implementation (Platt \& Glimcher, 1999; Louie, Grattan, \& Glimcher, Reward value-based gain control: divisive normalization in parietal cortex, 2011) (see (Kable \& Glimcher, 2009) for a review). Blood oxygen level dependent (BOLD) fMRI recorded during decision making supports these findings and show that subjective risk value correlates with activity in the IPFC (Tobler, O'Doherty, Dolan, \& Schultz, 2007). Specifically, lPFC activity to risk depends on risk attitude (Tobler, O'Doherty, Dolan, \& Schultz, 2007; Tobler, Christopoulos, O'Doherty, Dolan, \& Schultz, 2009; Holper, Wolf, \& Tobler, 2014) and this link causally contributes to risk attitude-dependent decisions (Knoch, et al., 2006; Fecteau, Knoch, Fregni, Sultani, Boggio, \& Pascual-Leone, 2007). There are also correlations between the subjective value of non-risky or risky choice options and neural activity in the striatum and ventromedial prefrontal cortex (Kable \& Glimcher, 2009; Bartra, McGuire, \& Kable, 2013; Levy, Snell, Nelson, Rustichini, \& Glimcher, 2010). In risk-averse participants, stronger IPFC activity to safer options is 
proposed to function as a "safety" signal, by playing an inhibitory role (Christopoulos, Tobler, Bossaerts, Dolan, \& Schultz, 2009). Consistent with the safety signal interpretation, Holper and colleagues (Holper, et al., 2017) suggested that demands for active working memory maintenance hinder the normal functioning of inhibitory mechanisms in the PFC. In contrast to activity in IPFC, activity in anterior cingulate cortex (ACC) increases with risky choice probability in risk-averse participants (Christopoulos, Tobler, Bossaerts, Dolan, \& Schultz, 2009), whereas risk perception has been associated with activity in the insula (Bossaerts, 2010). Thus, differences in behavior in risky decision making appear to arise from the interplay of several brain areas. However, little is known about the temporal dynamics of these activations.

A precise description of the temporal dynamics of brain activity would allow us to separate early processes, which are typically more automatic and sensory in nature, from late processes, which are more controlled (Bargh \& Chartrand, 1999). If behavioral differences among participants arise from the adoption of automatized, stereotypical heuristics, they may involve the use of highly-trained mathematical computations affording rapid access to solutions stored in long-term memory (LTM, see Logan's instance theory, (Logan, 1988)). This rapid memory access may be reflected in neural activity differences that should be apparent earlier in time than if they depend on later controlled processes (in Logan's terminology, algorithmic-based processing). In this study, we recorded ERPs, taking advantage of their millisecond temporal resolution, and investigated individual differences in early and late phases of the activity associated with risk-related decisions. 
Decision-making can be divided into multiple stages (Rangel, Camerer, \& Montague, 2008): 1) a perception phase, where information is processed and integrated (e.g., identification and representation of stimulus features and available actions); 2) a valuation phase (e.g., assigning value to possible options); 3) a decision phase (informed by valuation); 4) a selection phase (reporting the choice) and 5) outcome appraisal or feedback evaluation. Most ERP studies in risky decision making have focused on outcome-appraisal (Polezzi, Sartori, Rumiati, Vidotto, \& Daum, 2010; Schuermann, Endrass, \& Kathmann, 2012; Zhang, et al., 2014; Zheng, Li, Wang, Wu, \& Liu, 2015; Xu, Pan, Wang, Spaeth, Qu, \& Rao, 2016; Fernandes, et al., 2018) or reward anticipation (Zheng, Li, Wang, Wu, \& Liu, 2015; Zheng, An, Li, \& Xu, 2020).

In this study, we focused on the perception, valuation and decision phases using the stimuluslocked ERPs that follow the presentation of options and precede participant's choice selection. We first measured the parietal N1 component (Eimer, 1998; Hillyard, TederSälejärvi, \& Münte, 1998), also termed N170 (Emmorey, Midgley, Kohen, Sehyr, \& Holcomb, 2017), N180 or N2, to get an index of attention. The P200, with a latency of about 200 ms after stimulus onset, is supposed to reflect automatized phases of stimulus evaluation (Luck \& Hillyard, 1994; Testa, et al., 2020), including rapid access to LTM representations, and therefore be related to choice consistency across trials. ERP components that have been analyzed with regard to risk-taking include the medial frontal negativity (MFN, or frontocentral N2), a negative component peaking 250-300 ms after stimulus onset (Gehring \& Willoughby, The medial frontal cortex and the rapid processing of monetary gains and losses, 2002) and the mid-latency P3, P300, or late positive potential (LPP). The stimulusrelated MFN may be considered as part of a family of ERP components that includes the 
feedback-related negativity (FRN) and the error-related negativity (ERN) (Chandrakumar, Feuerriegel, Bode, Grech, \& Keage, 2018), which are elicited in conditions in which stimulus or response conflict is present. These components are all thought to derive from the activation of the ACC, which may be associated with the perceived need by the participant to exert cognitive control in response to stimulus/contextual conditions (Botvinick, Braver, Barch, Carter, \& Cohen, 2001). We expect therefore the amplitude of MFN to be indexing the use of controlled processing and therefore to be associated with a non-automatized, less consistent behavior. The P300 component is proposed to reflect the end of the evaluation process (Coles, Gratton, Bashore, Eriksen, \& Donchin, 1985), and its amplitude may reflect subjective uncertainty (Donchin \& Coles, 1988). However, the P300 is also sometimes difficult to dissociate from another brain activity associated with the processing of emotional stimuli, the LPP (see (Hajcak, Dunning, \& Foti, 2009)), so in this paper we will use the term P300/LPP to indicate a complex of activity encompassing them both. When evoked during outcome appraisal, the P300/LPP has been associated with the motivational significance of the outcome (Nieuwenhuis, Aston-Jones, \& Cohen, Decision making, the P3, and the locus coeruleus-norepinephrine system, 2005), an association enhanced by risk-taking behavior (Polezzi, Sartori, Rumiati, Vidotto, \& Daum, 2010).

Here, we are interested in characterizing the neuroscientific processes underlying the perception (early ERPs), valuation (mid-latency ERPs) and decision (late latency ERPs) processes of risky choice options and relate them to individual differences in risk taking and choice inconsistency. We expect the degree of choice consistency to modulate ERP components associated with automatized stimulus processing, such as the anterior P200. We also expect the MFN to play a key role in the valuation process and to be associated with 
individual differences in behavior. Finally, we expect the late part of the ERP to be associated with the subsequent choice selection, an association that could be modulated by risk aversion, because the motivational significance of a choice under risk is likely to vary across individuals with different attitudes towards risk.

\section{Methods}

\section{Participants}

Twenty-four healthy adults (13 females, age range 27-61 years, 23 right-handed) took part in the experiment. Prior to the experiment, participants were screened in order to exclude people with severe sleep disorders (Buysse, Reynolds 3rd, Monk, Berman, \& Kupfer, 1989) because of a collateral interest in sleep effects on decision making, not included in the current study. Participants had normal or corrected-to-normal vision and no history of psychiatric (Spitzer, Kroenke, Williams, \& Löwe, 2006; Kroenke, Spitzer, \& Williams, 2001) or neurological disorders. Two right-handed participants were excluded from the analysis because it was not possible to compute some of the behavioral measurements in some conditions (more details in the Behavioral measurements subsection). Therefore, the final sample comprised 22 participants (women $=12$, mean age $=38.6, \mathrm{SD}=10.5$, range $27-61,21$ right-handed). Prior to participation, all subjects were informed about the procedures and provided signed informed consent. This project was approved by the Cantonal Ethic Research Committee (CCER) of Geneva (2019-00095).

\section{The risky decision task}

We used a risky decision task similar to one used previously (Tobler, O'Doherty, Dolan, \& Schultz, 2007; Tobler, Christopoulos, O'Doherty, Dolan, \& Schultz, 2009; Christopoulos, 
Tobler, Bossaerts, Dolan, \& Schultz, 2009; Holper, Wolf, \& Tobler, 2014; Holper, et al., 2017). The task consisted of 20 blocks of 20 trials each; on each trial, participants were presented with a choice between two options (left or right, Figure 1). One option had two possible outcomes, each with a probability of $50 \%$. The difference between the two possible outcomes defined the objective risk-level associated with that option (Tobler, O'Doherty, Dolan, \& Schultz, 2007; Tobler, Christopoulos, O'Doherty, Dolan, \& Schultz, 2009; Christopoulos, Tobler, Bossaerts, Dolan, \& Schultz, 2009; Holper, Wolf, \& Tobler, 2014; Holper, et al., 2017) . This "risky" option encompassed two risk levels (small, large) with corresponding EVs. For the small EV (30) the possible outcomes were 15 and 45 (lower risk) or 10 and 50 (higher risk). For the large EV (60), the possible outcomes were 40 and 80 (lower risk) or 30 and 90 (higher risk). The other option was always free of risk (i.e., the two possible outcomes had the same value; "safe" option). For each risky alternative, the set of possible EVs of the safe option were distributed within the range of the two outcomes according to a pseudo-normal distribution centered on the average certainty equivalents, identified in previous research (Holper, et al., 2017). For each block, we randomly presented one of the 4 possible risky alternatives. In each trial, we randomly presented one of the possible safe options associated with the current risky alternative.

Participants sat in a comfortable chair and received written task instructions. Participants were also informed that one trial out of all 400 trials would be randomly selected, and that they would receive $10 \%$ of the outcome of that selected trial at the end of the experiment.

The experiment was run with the lights off, and the computer screen background was dark grey. Each trial began with participants pressing the space bar. Next, they were asked to fixate 
on a white cross at the center of a computer screen placed at $65 \mathrm{~cm}$ distance. Options were presented to the left and right of the fixation cross with a lateral visual angle of 5.27 degrees and vertical visual angle of 3.74 degrees. The side at which the safe and risky options were presented, as well as their vertical placement (i.e., top or bottom) were randomly selected. To avoid learning effects, no feedback about the outcome of the risky option was given to the participants. However, the fixation cross turned purple when a choice was made. The intertrial interval was randomly distributed between $0.5-1.5 \mathrm{~s}$, to avoid cross- and within-trial contamination of EEG signals.

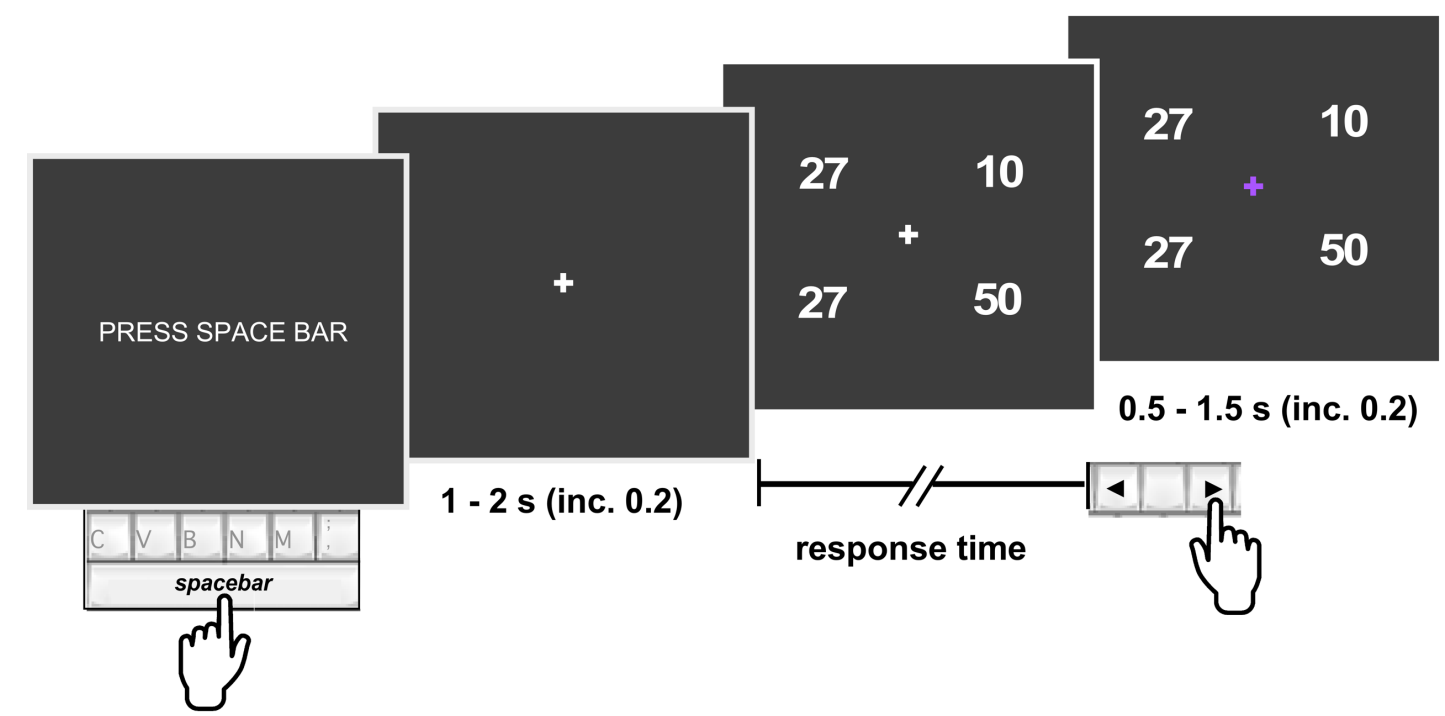

Figure 1: Schematic representation of a trial. Each trial started when the participant pressed the spacebar. A fixation cross was presented for 1-2 s, with duration varying in fixed intervals of $200 \mathrm{~ms}$, followed by the presentation of options. Participants were asked to make a choice by pressing the left or right arrow key on the computer keyboard with their right index finger. The fixation cross turned purple after the choice was made.

\section{Electrical recording}

The EEG was recorded continuously at a $1024 \mathrm{~Hz}$ sampling frequency (24 bit resolution) with an ActiveTwo MARK II Biosemi EEG System (BioSemi B.V., Amsterdam, The Netherlands) using NeuroSpec Quick Caps with 64 scalp Ag/AgCl active electrodes (extended 10/20 
layout). Two pairs of bipolar electrodes were used to record vertical and horizontal eye movements. The EOG electrodes were placed at the outer canthi of both eyes and above and below the left eye. EEG and EOG were recorded with a band-pass filter of 0.05-200 Hz. Impedance was kept below $5 \mathrm{~K} \Omega$. Scalp electrodes were referenced to the left mastoid during recording and re-referenced off-line to the average of the left and right mastoid.

\section{EEG data pre-processing and analysis}

EEG data were preprocessed and analyzed with the ERPLAB toolbox (Lopez-Calderon \& Luck, 2014), and with dedicated MATLAB scripts (MATLAB, The MathWorks, Inc). EEG data were segmented into epochs of $800 \mathrm{~ms}$ (baseline $=160 \mathrm{~ms}$ ) based on markers identifying the start of the stimulus, i.e. the presentation of the options. To minimize the contamination from the response-induced activity on the ERPs trials with response times shorter than the ERP response window, i.e. 640 milliseconds (ms), were excluded. Trials with response times longer than 6 seconds were also excluded from further analysis (mean rejected $=0.06, \mathrm{SD}=$ 0.01). Ocular artifacts (blinks and saccades) were removed from the signals by means of the eye movement correction procedure (EMCP) (Gratton, Coles, \& Donchin, 1983). In addition, epochs were visually inspected for contamination by muscular artefacts and other recording artifacts and excluded from further analyses if those artifacts were detected. Epochs passing all the exclusion criteria (mean $=76 \%$ ) were low-pass filtered at $40 \mathrm{~Hz}$. Average ERPs waveforms were derived for each subject, electrode and condition.

As mentioned in the introduction, here we focused on the perception and valuation phases of the study. We defined 3 clusters of frontocentral electrodes (midline: Fz, F1, F2, FCz, FC1, FC2; left: F3, F5, F7, FC3, FC5, FT7; right: F4, F6, F8, FC4, FC6, FT8) and 3 clusters of centro- 
parietal electrodes (midline: Pz, P1, P2, CPz, CP1, CP2; left: P3, P5, P7, CP3, CP5, TP7; right: P4, P6, P8, CP4, CP6, TP8). For each participant, the mean amplitude of each ERP component was computed for each of the 4 experimental conditions at the 3 groupings (midline, right and left) of the relevant measurement cluster for that component (12 observations per participant). Poor ERP signals for each condition from each cluster were identified using the average standardized measurement error (SME) (Luck, Stewart, Simmons, \& Rhemtulla, 2021) of the individual electrodes that compose the cluster. Clusters with an average SME > 5 microvolts $(\mu \mathrm{V})$ were excluded from further analyses (mean rejected $=0.05$ for frontal signals; mean rejected $=0.08$ for parietal signals). In addition, the observations from one participant in one condition (risk low and $\mathrm{EV}_{\text {high) }}$ were identified as outliers and were excluded from further analysis.

For each participant and condition, we measured the parietal N1 as the mean amplitude between 150-250 ms after stimulus onset at the centro-parietal electrode sites. This time window was centered around the component's mean latency at electrode site Pz (mean = 197.78, $s d=17.50$ ). Following Wang and colleagues (Wang, Li, Wang, Zhu, Pan, \& Li, 2019), we measured the P200 as the mean amplitude between 150-250 ms and the MFN as the mean amplitude between 250-350 ms at fronto-central clusters. We measured the P300/LPP at the centro-parietal clusters as the mean amplitude between 300-600 ms (Klein, Coles, \& Donchin, 1984).

We first examined the effects of conditions on the components' mean amplitude. A three-way (EV level, Risk level and cluster position) ANOVA was performed for each ERP component. Secondly, we aimed at identifying significant interactions between components and 
individual differences in behavior. An ordinary least squares method was used to analyze the relationship between individual differences in behavior $\left(K_{\text {mean }}\right.$ and $\left.R A_{\text {mean }}\right)$ and experimental conditions (EV and risk level) and ERP components. Because there may be age and gender differences in attitude towards risk (Borghans, Heckman, Golsteyn, \& Meijers, 2009; Zilker, Hertwig, \& Pachur, 2020), we also examined the effects of these demographic variables in our analyses. Specifically, age, gender (male $=1$, female $=2$ ) and the position of the cluster of electrodes (medial, left and right) were entered as predictors in the multiple regression model. Standardized coefficients and partial correlations were computed using robust standard errors (summ, R function from CRAN jtools package (Long, 2021)). Significant interactions between behavioral measurements and ERP components were further analyzed for correlation with between conditions differences.

For each individual, the decision-level component, i.e. P300/LPP mean amplitude, was computed for each 2 subsequent decisions, i.e. risky and safe choices, at each of 3 cluster position ( 6 observations per participant). Clusters with an average SME $>5$ microvolts $(\mu \mathrm{V})$ were excluded from further analyses (mean rejected $=0.03$ for frontal signals; mean rejected $=0.07$ for parietal signals). A two-way (cluster position, decision) ANOVA was performed on each ERP component. Significant interactions between behavioral measurements and the ERP component found in the regression analysis were further analyzed for correlation with between decisions differences. Biweight midcorrelation (bicor, $\mathrm{R}$ function from CRAN WGCNA package (Langfelder \& Horvath, 2008)) was used for all correlation tests. In a further analysis, presented in Supplementary materials, we examined whether excluding participants with a $R A_{\text {mean }}<-1$ (which could be considered as "risk seeking" individuals) 
might influence the regression result. Supplementary materials also report an exploratory group analysis.

\section{Behavioral measurements}

Following previous research (Christopoulos, Tobler, Bossaerts, Dolan, \& Schultz, 2009), we calculated attitude towards risk from the choices made in the task. Specifically, for each option we computed the certainty equivalent (CE), which is the outcome of the safe option for which the participant is indifferent (i.e., equally likely to choose) between the safe and the risky option (Luce, 1999) (see Figure 2 for an example). To determine CEs, we specified a pseudo sigmoid function (Strasburger, 2001) and computed the point on the x-axis for which $f(x)=0.5$ (red square in Figure 2). The pseudo sigmoid function (red line in Figure 2), was fitted to the choice data with the following specification:

$f(x)=\frac{1}{1+e^{-k *\left(x-x_{0}\right)}}$

where $\mathrm{x}$ takes only values within the range of outcomes of the risky option, $x_{0}$ is the minimum outcome and $\mathrm{k}$ is the slope parameter of the sigmoid function. We converted estimated slope parameters into degrees with the following transformation:

$K=k * \frac{180}{\pi}$

The maximal value of $\mathrm{K}$ is $90^{\circ}$ and corresponds to a special case without inconsistency, such that only a threshold function could fit the data.

As mentioned earlier, two participants were excluded from further analysis because, in at least one condition, they hardly ever chose one of the two options, such that the sigmoid function fitting procedure was not possible. Risk attitude was defined by the difference 
between the CEs of the high- and the low-risk conditions, keeping EV constant (Christopoulos, Tobler, Bossaerts, Dolan, \& Schultz, 2009). Thus, larger positive (or negative) differences correspond to increasing risk aversion (or risk proneness). Risk aversion (RA) was computed for the two levels of $\mathrm{EV}$ (i.e., $\mathrm{EV}=30$ and $\mathrm{EV}=60$ ). Both RA measures were standardized to the low EV condition in order to be comparable. We then computed their mean $\left(R A_{\text {mean }}\right)$ and the absolute difference between both values $\left(R A_{\text {diff }}\right)$. The slope parameter of the S-curve was estimated for each condition and converted into degree angle $(\mathrm{K})$. The mean of $\mathrm{K}$ values across the 4 conditions $\left(K_{\text {mean }}\right)$ was used as a measure of withincontext consistency: the smaller the K, the more variable the observed choices.
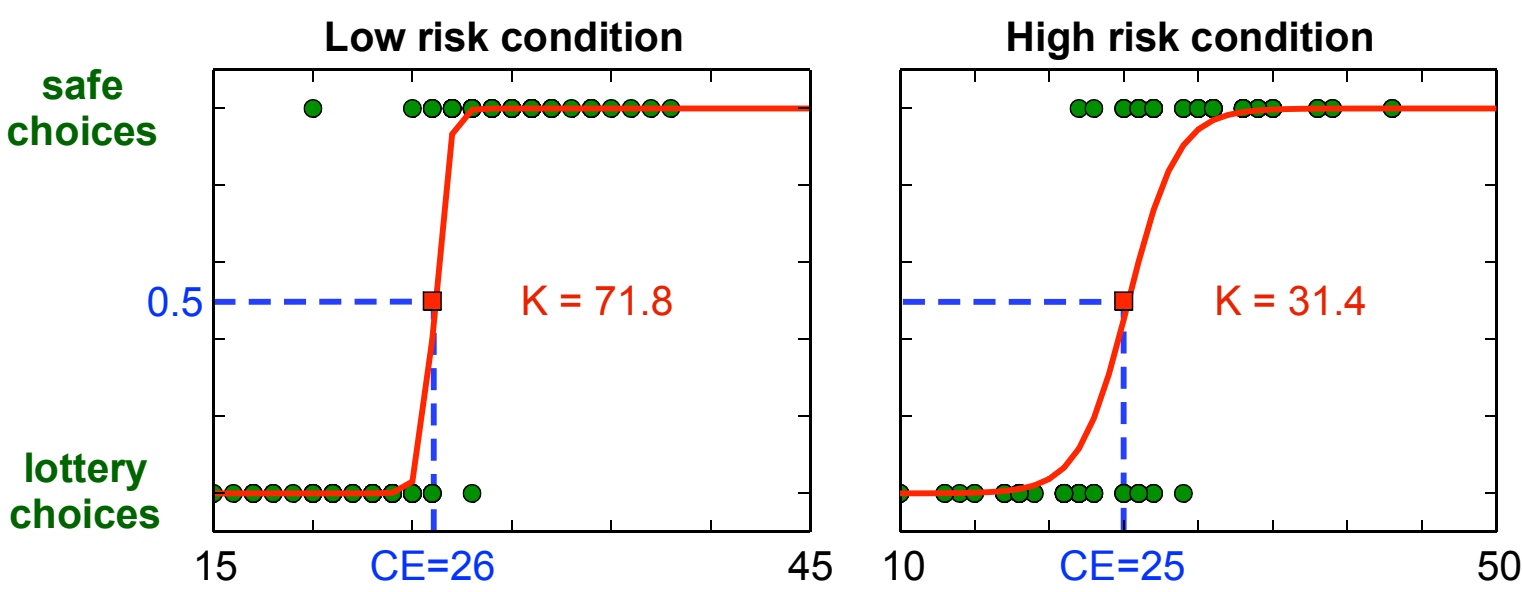

Figure 2: Example behavior of a participant. The left and right panels illustrate choices (green dots) in the low (i.e., 15, 45) and high (i.e., 10, 50) risk conditions, respectively. The $x$-axis represents the outcome of the safe option. 


\section{Results}

\section{Behavior}

Overall, behavioral results converge with those of previous studies (Holper, et al., 2017). As shown in Figure 3A the percent of risky choices decreased as the value of the safe option increased for all conditions. The average RT across participants ranged from 1035 to 3237 ms $($ mean $=1906, s d=561)$. Figure 3B shows that response times followed an inverse Ushaped relationship based on the safe option value. This suggests that participants experienced a higher level of conflict (and therefore were slower to respond) when faced with choices that were close to their behavioral inflection point. For all conditions, response times were, on average longest for the safe option, with values around the average CE (Figure 3C). No significant correlation was found between $R A_{\text {mean }}$ and the average response time (coefficient $=0.10, p=.65$ ) or between $K_{\text {mean }}$ and the average response time (coefficient $=-$ 0.16, $\mathrm{p}=.48$ ). Consistently with previous studies (Tobler, Christopoulos, O'Doherty, Dolan, \& Schultz, 2009; Holper, Wolf, \& Tobler, 2014; Holper, et al., 2017) we observed that most participants were risk averse (Figure 3D).

To summarize, the overall behavior of our sample was consistent with previous studies. However, the measurement of $R A_{\text {diff }}$ and $K_{\text {mean }}$ allowed us to observe some heterogeneity within our sample, where subgroups could be identified. Exploratory analyses reported in Supplementary Materials, based on a more fine-grained grouping of the participants, suggest that they may differ in early visual attention mechanisms. 

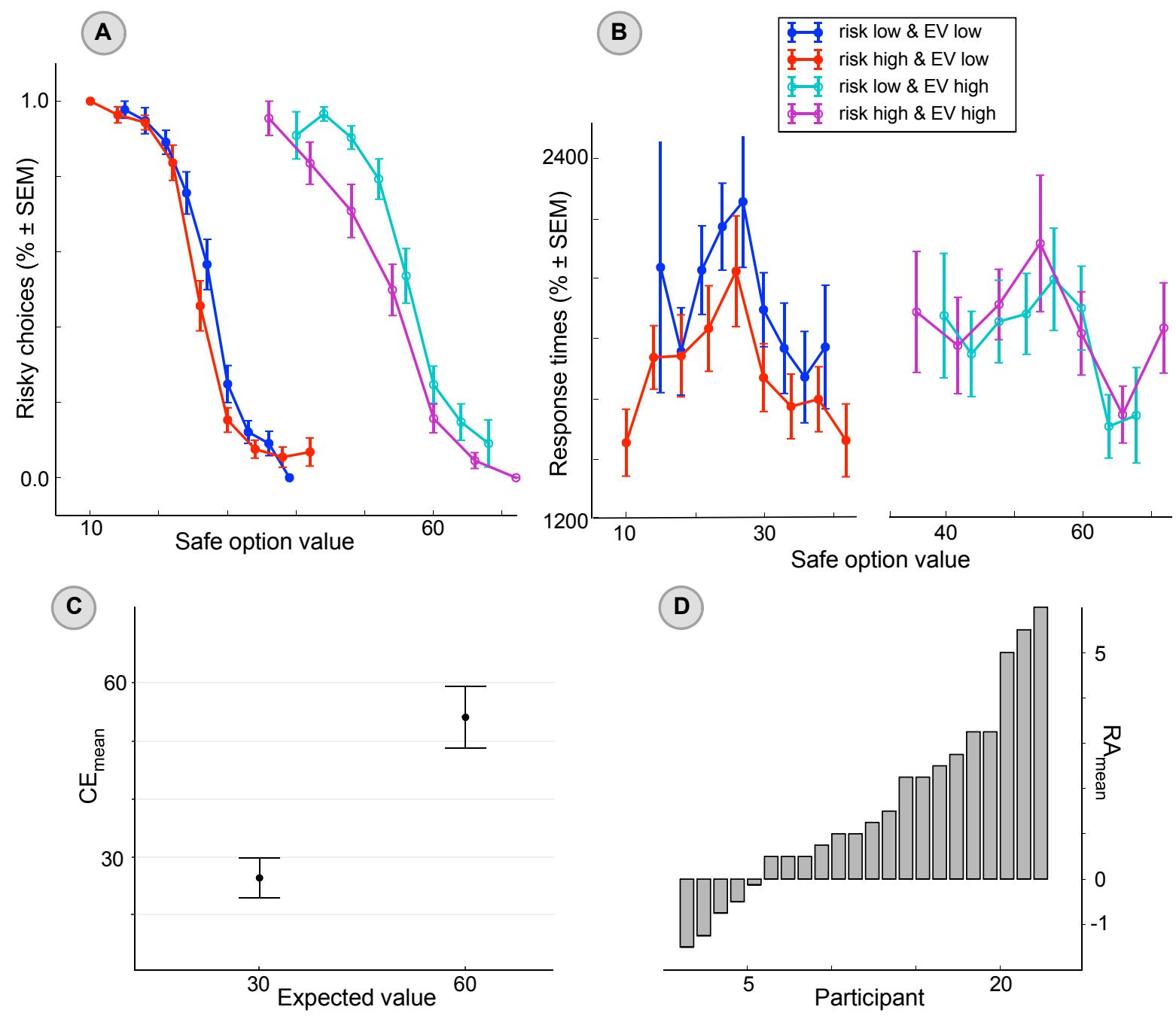

Figure 3: Behavioral results (N=22). A. Percent risky choices as a function of risk-free option values across the 4 conditions. Results are smoothed by averaging risk-free option values at evenly-spaced points on the $x$-axis. B. Response times as a function of risk-free option values across the 4 conditions. $C$. Average certainty equivalents for low and high expected value (mean $\pm \mathrm{sd}$ ). C. Risk aversion mean for each participant.

\section{ERPs}

Here we present two distinct ERP analyses both locked to the presentation of the options to the participant. First, we sorted ERP activity separately for each stimulus condition in order to analyze ERP correlates of objective changes in EV and risk levels, as well as their subjective modulations characterized by individual differences in risk aversion $\left(R A_{\text {mean }}\right)$ and within- 
bioRxiv preprint doi: https://doi.org/10.1101/2021.05.10.443362; this version posted May $10,2021$. The copyright holder for this preprint

(which was not certified by peer review) is the author/funder, who has granted bioRxiv a license to display the preprint in perpetuity. It is made available under aCC-BY-NC-ND 4.0 International license.

condition consistency $\left(K_{\text {mean }}\right)$. Second, we sorted the ERP activity as a function of the subsequent decision for risky or safe choices.

\section{ERP results separated by stimulus condition}

For this analyses, trials were sorted in four types, based on the EV (high or low EV, expected to impact motivational level) and the possible range of rewards associated with making a risky choice (larger and small ranges, expected to high and low "risk" conditions, expected to impact outcome uncertainty). The grand average waveforms for the six electrode clusters based on all participants in the study are presented in Figure 4. 


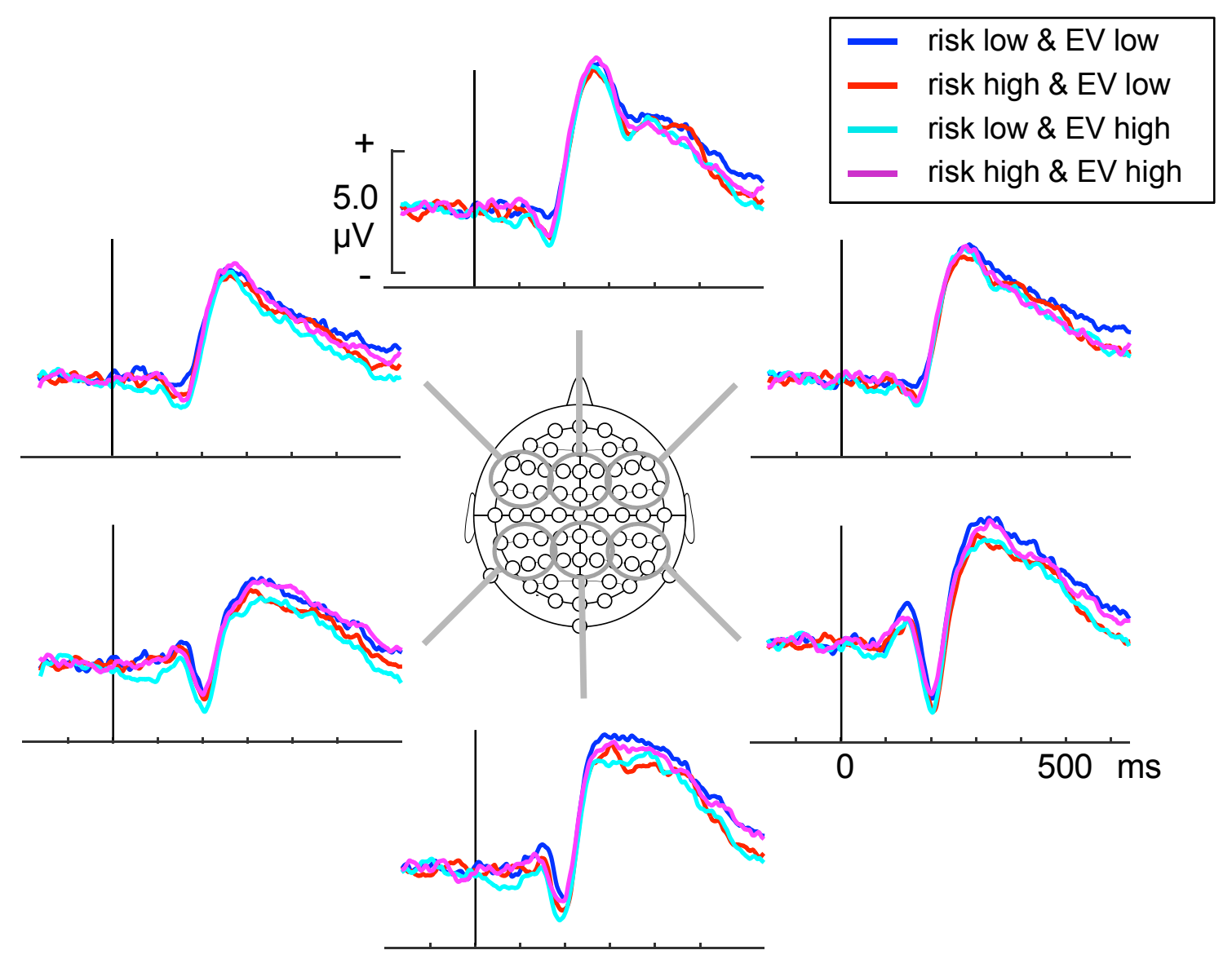

Figure 4. Grand average ERP waveforms from the six electrode cluster for each trial type. The set of electrode locations used for each cluster are indicated in the diagram at the center of the figure.

The peak amplitude values for the various ERP components for each participant were computed using the procedures described in the Method section, and entered into a factorial ANOVA with 3 factors: Electrode cluster position, EV, Risk. The mean (and standard deviations) of the components amplitudes can be found in Table 1. The results of this analysis indicated a significant main effect of cluster position for MFN and the P300/LPP had (MFN: $F_{(2,235)}=3.19, p=.04$; P300/LPP: $\left.F_{(2,227)}=12.15, p<.01\right)$. Tukey tests indicated that the MFN was larger at fronto-central locations, and the P300/LPP was larger at centro-parietal conditions. Both phenomena are consistent with what is known regarding these ERP components. 
However, there were no significant main effects or interactions related to EV or risk levels for any component.

\section{Relationships between ERPs and risk tolerance and choice consistency}

The main focus of this paper is the investigation of the relationship between individual differences in behavior during risky decision making (which we expect to reflect the use of different types of heuristics - which we can label as "automatized" and "fuzzy", respectively) and the brain dynamics occurring while making these choices, as identified using ERPs. As delineated in the Introduction, we expect the use of these different heuristics to affect ERP activity occurring at different latencies: early latency (i.e., $<250 \mathrm{~ms}$ ) ERP activity may reflect attention levels as well as the use of automatized computational processes (which should be larger for individuals using "automatized" heuristics), middle latency (between 250 and 400 ms) ERP activity may reflect "controlled" valuation processes (mid-latency) which would be particularly critical when the choice between options is not obvious, and long latency $>400$ ms) ERP activity which may reflect subjective uncertainty about the outcome of a particular choice (we expect both middle and long latency ERP activity to be larger in individuals using "fuzzy" heuristics). To assess these individual differences in behavior, we characterized individual differences behaviorally using the risk aversion $\left(R A_{\text {mean }}\right)$ and within-condition consistency ( $\left.K_{\text {mean }}\right)$ parameters, which were estimate using the procedures described above. We then used ordinary least squares regression to assess how these behavioral measures relate to ERP components. In this regression model we also introduced other variables (such as age and sex) that are known to affect risky choice behavior as additional predictors. 
The beta weights indicating the relationship between these demographic and behavioral variables and ERP parameters are presented in Table 2. Significant relations between an ERP component and a behavioral measure were further investigate using robust bi-weighted midcorrelation analyses (presented in Table 3). These results are generally consistent with the idea that individual differences in behavior during risky choices may be associated with different brain dynamics that are revealed by ERP components.

To provide a graphical description of how these relationships explicate in producing different type of ERP waveforms, participants were sorted into groups based on their behavioral parameters, and grand average ERP waveforms were calculated for each group (see Figure 5A). In the remainder of this section we describe the relationships between behavioral and the amplitude of specific ERP components, ordered by latency.

We also repeated some of the analysis excluding two individuals whose $R A_{\text {mean }}$ score indicated a clear "risk-seeking" behavior $\left(R A_{\text {mean }}<-1.0\right)$, since it is possible that, for these participants, uncertainty might not have emotionally negative consequences. If this were the case, the relationship between using an inconsistent ("fuzzy") heuristic and emotional activation might break down.

\section{Parietal N1 component}

The amplitude of the parietal N1 is influenced by attention factors (Eimer, 1998; Hillyard, Teder-Sälejärvi, \& Münte, 1998). We did not have specific predictions about how attention factors might relate to the adoption of different heuristics during risky decision making. Table 2 reports no significant interaction between $R A_{\text {mean }}$ or $K_{\text {mean }}$ and the parietal N1 mean 
amplitude. The proportion of the variance in the N1 mean amplitude that is predicted from the independent variables is small as compared to the other dependent variables (adjusted $\mathrm{R}^{2}=.08$ for $\mathrm{N} 1$; adjusted $\mathrm{R}^{2}=.23$ for $\mathrm{P} 200$; adjusted $\mathrm{R}^{2}=.26$ for MFN; adjusted $\mathrm{R}^{2}=.16$ for P300/LPP). This suggests that there may not have been clear differences in the level of attention paid to the stimuli as a function of the adoption of different behavioral strategies (or heuristics).

\section{Frontal P200 component}

The frontal P200 may reflect the adoption of automatized computations which may make behavioral choices more consistent across trials (Testa, et al., 2020; Luck \& Hillyard, 1994), and therefore should be larger for individuals with high $K_{\text {mean }}$ (and low $R A_{\text {mean }}$ ). The average ERP waveforms (Figure 5A) differed between strictly risk averse and risk neutral individuals at the medial fronto-central cluster. This visual impression is consistent with a significant correlation between the P200 mean amplitude and $R A_{\text {mean }}$ (shown in Table 1). The more risk averse the participant, the more negative was the P200 (standardized coefficient $=-0.84$, $p<.001$ ). In other words, the more positive this component, the more risk tolerant participants were, in line with subjective risk value.

Moreover, the more consistent the individual $\left(K_{\text {mean }}\right)$, the higher the P200 mean amplitude (standardized coefficient $=0.41, p<.001) . R A_{\text {mean }}$ and $K_{\text {mean }}$ regression results were robust to: 1) the exclusion of the two risk seeking participants. 2) the exclusion of individuals belonging to the inconsistent group 2 (cf. Supplementary materials). In the absence of risk seeking participants, low risk aversion corresponds to risk neutrality. Thus, our results suggest that P200 is higher in individuals who are more rational in its simplest economic 
definition, i.e. individuals who are maximizing the expected value of outcomes. These findings are consistent with the hypothesis that the P200 may reflect the adoption of automatized computations which may make behavioral choices more consistent across trials.

We further broke down this relationship for different types of trials. $K_{\text {mean }}$ was positively correlated with the difference in frontal P200 mean amplitude at the medial fronto-central cluster between high and low EV in the low risk level condition (coefficient $=0.48, p<.05$; Figure 5B; Table 2). In other words, at low risk level, the P200 showed a relation between value sensitivity and consistency. This relation was not observed with high risk level. At least at low risk level, the effect of EV on the P200 appears to relate to individual consistency; however, it is possible that under high motivational stress, this relationship might weaken.

\section{MFN component}

The MFN may be related to a perceived need for control which may arise under conflict or uncertainty conditions (see (Botvinick, Braver, Barch, Carter, \& Cohen, 2001)), and therefore should be larger for individuals with larger $R A_{\text {mean }}$. Correspondingly, in Figure $5 \mathrm{~A}$, the MFN component appears to differ between risk averse and risk neutral individuals. Indeed, the more risk averse the participant, the more negative the MFN (Table 1; standardized coefficient $=-1.19, p<.001)^{1}$. In line with an impact of risk attitude on the MFN, we found a significant correlation between $R A_{\text {mean }}$ and the difference in MFN mean amplitude between

\footnotetext{
${ }^{1}$ Statistically, these results were robust to: 1) the exclusion of the two risk seeking participants and 2) the exclusion of the individuals in inconsistent group 2 (cf. Supplementary materials). Together with the larger, i.e. more negative, MFN signal for risk averters, these results are consistent with a risk perception signal.
} 
the high and low risk level conditions for low EV at the medial fronto-central cluster (coefficient $=0.44, p<.05$; Table 2). Figure 5C indicates that the MFN was more negative in the high-risk level condition for participants with low risk aversion, but only in conditions with low EV. Thus, as for the P200 component, the effects of behavioral strategy appear more evident at lower motivational levels.

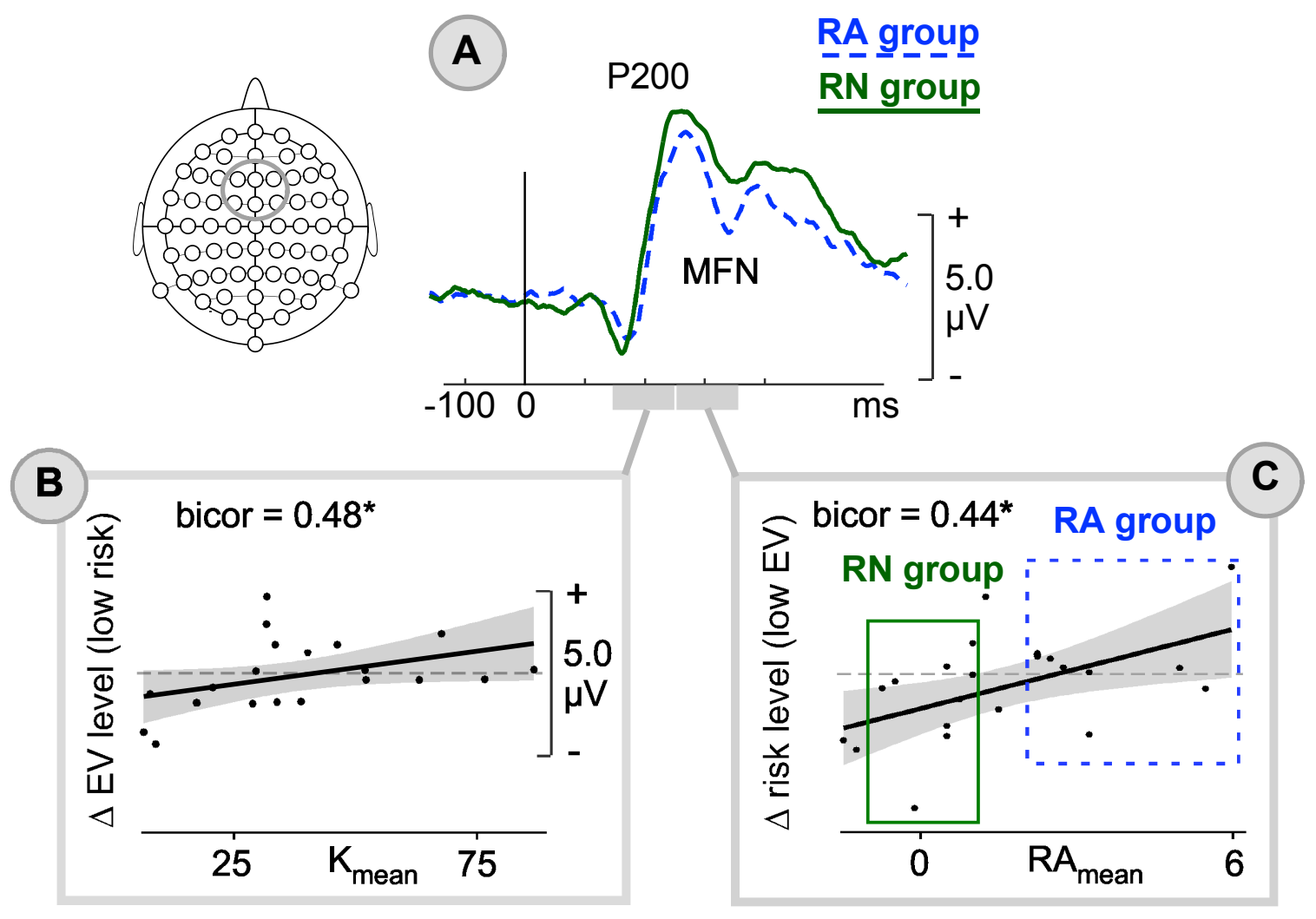

Figure 5: Relationship between risk attitude and ERP activity. A. Grand average ERP waveforms at the medial fronto-central cluster across all conditions; separate waveforms were computed for strictly risk averse ( $R A$ in blue, $R A_{\text {mean }}>2$ and $R A_{\text {low } E V}>0$ and, $R A_{\text {high } E V}>0, N=9$ ) and risk neutral participants $\left(R N, \operatorname{abs}\left(R A_{\text {mean }}\right)<1, N=9\right)$. B. Correlation of the difference between the P200 mean amplitude in the high - low EV level condition for low level of risk at the medial fronto-central cluster (y-axis) with $K_{\text {mean }}$ ( $x$-axis). C. Correlation of the difference between the MFN mean amplitude in the high - low risk level condition for low level of risk at the medial fronto-central cluster (y-axis) with $R A_{\text {mean }}(x$-axis). For $B$. and D., robust biweight midcorrelation coefficient (bicor) is specified for each plot. ${ }^{*} p<.05$. 


\section{P300/LPP component}

The P300/LPP component (which tend to be larger at centro-parietal locations) has been associated with uncertainty about outcomes and emotional involvement with the stimulus conditions (e.g., Hajcak et al., 2009), which should be greater in individuals using fuzzy heuristics and showing high $R A_{\text {mean }}$. Correspondingly, robust regression analyses indicated that the mean P300/LPP amplitude was modulated by $R A_{\text {mean }}$ and cluster position (Table 2). This component was more positive at the medial centro-parietal cluster as compared to the left (coefficient $=-1.54, p<.001$ ) and to the right (coefficient $=-0.63, p<.05$ ) centroparietal clusters. More importantly, the more risk averse participants were, the more positive was the P300/LPP component (coefficient $=0.49, p<.001$ ). The exclusion of risk-seeking participants impacted the coefficient of $R A_{\text {mean }}$ (coefficient $=0.07, p>.05$, in Supplementary materials). This may suggest that the association between $R A_{\text {mean }}$ and a reduced mean amplitude of the P300/LPP may, at least in part, be due to the negative emotional consequences of outcome uncertainties in risk-averse individual. Further research with more risk-seeking participants is needed to obtain a clearer demonstration that the relationship between $R A_{\text {mean }}$ and P300/LPP differ between risk-averse and risk-seeker individuals.

\section{ERP sorted based on the participants' decisions}

We were also interested in the brain processes leading participants to choose the uncertain (risky) option or the certain (safe) option on any particular trial. Therefore, we sorted trials according to the decision made by the participants. For this analysis, we pooled the different 
trial types (high risk/high EV, low risk/high EV, high risk/low EV and low risk/low EV) together. The corresponding grand average waveforms at the parietal clusters are presented in Figure 6. A two-way repeated-measures ANOVA (with option choice and electrode cluster as factors) indicated no significant main effect of decision or any interactions with position (Table 1). Unsurprisingly, we replicated the electrode cluster effect on the amplitude of the P300/LPP component. We also performed an analysis to determine whether there was any data point at which the waveforms for the safe and risky decision differed. To this end, we used paired t-tests on the difference between risky and safe individual average waveforms at each of the 64 electrode sites, using a Bonferroni-Holm correction for multiple comparison. This analysis indicated a significantly greater positivity at $340 \mathrm{~ms}$ after stimulus onset in risky choice trials compared to safe decisions at several right and mid-parietal electrode sites. 
B
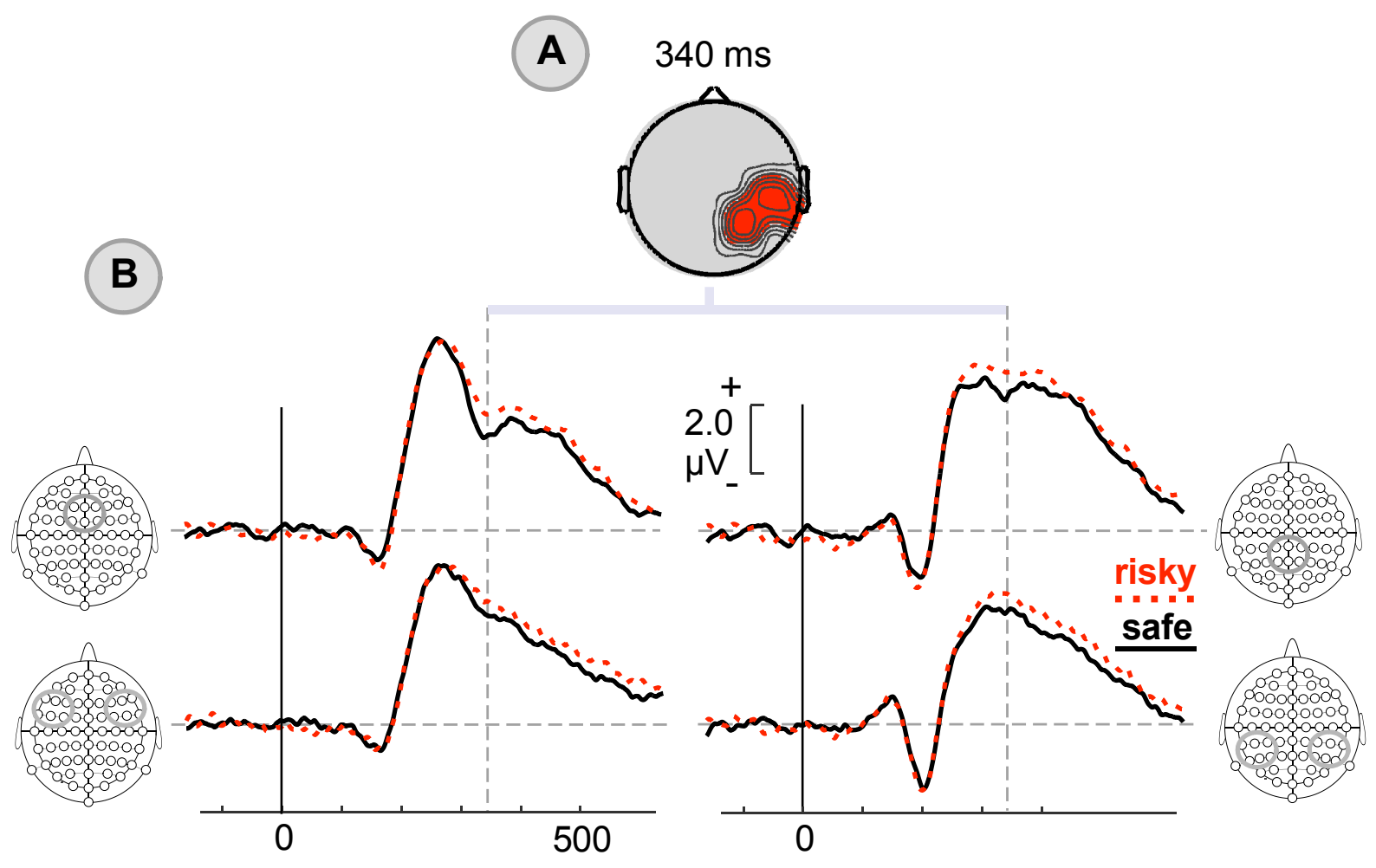

\section{Figure 6:}

A. topographic map of the significant difference between risky and safe choices at $340 \mathrm{~ms}$ ( $r e d=p<.05$; grey=p>.05). B. Grand average ERP waveforms separated by subsequent choices $(N=22)$ at medial clusters of electrodes (top) and lateral clusters (bottom: merging left and the right clusters). The frontal clusters (left) are shown separately from the parietal clusters (right).

As for the trial-type analyses reported above, we investigated whether there were individual differences linked to overall behavior patterns in the ERPs sorted based on the actual participants' decisions. Although analyses were conducted based on data from each individual participant, for illustration purposes grand average waveforms were calculated for individuals sorted into the same groups risk-averse (RA) and risk-neutral (RN) groups used for the trial-types analyses. Group average ERP waveforms for the right centro-parietal electrode cluster are presented in Figure 7. The results indicated that the P300/LPP at right parietal electrode sites differed depending on the partipants decisions, in line with the 
hypothesis that the P300/LPP entails behaviorally relevant information. Furthermore, we found a significant negative biweight midcorrelation for the difference in amplitude between risky and safe choices with $R A_{\text {mean }}$ in the right centro-parietal electrode cluster (coefficient $=-0.49, p<.05$ ). No significant correlation was observed for the left and medial centroparietal clusters (left: coefficient $=0.03, p=0.91$; medial: coefficient $=-0.11, p=0.63$ ). The correlation in the right centro-parietal cluster was robust to the exclusion of risk-seeking participants (coefficient $=-0.46, p<.05$ ). Hence, we find that lateralized parietal activation is behaviorally relevant, which is consistent with animal studies (Platt \& Glimcher, 1999; Louie, Grattan, \& Glimcher, Reward value-based gain control: divisive normalization in parietal cortex, 2011). This effect was dependent on risk aversion (even in the absence of risk-seeking participants): the lower the risk aversion, the higher the effect. Overall, risk aversion appears to modulate the late lateral parietal decision signal in a quasi linear fashion. 

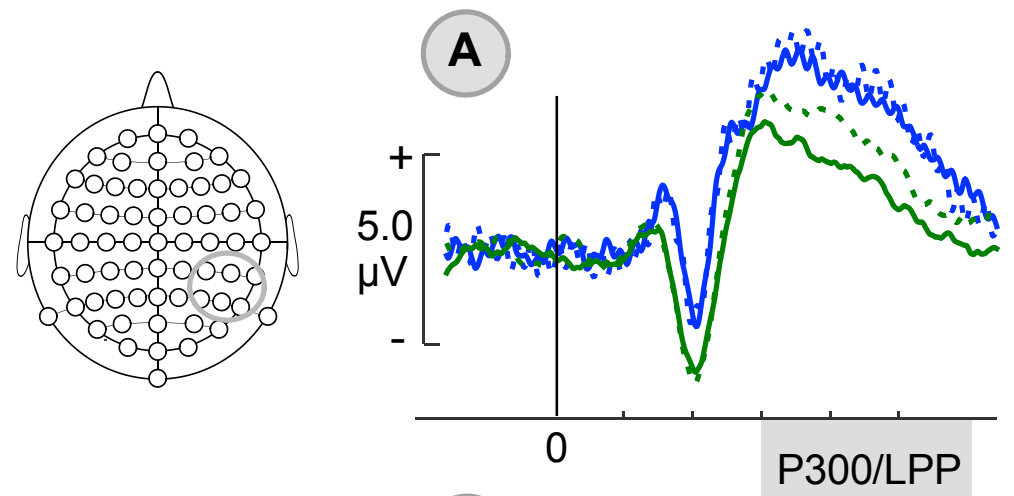

RA group: risky

RA group: safe

RN group: risky.

RN group: safe

B

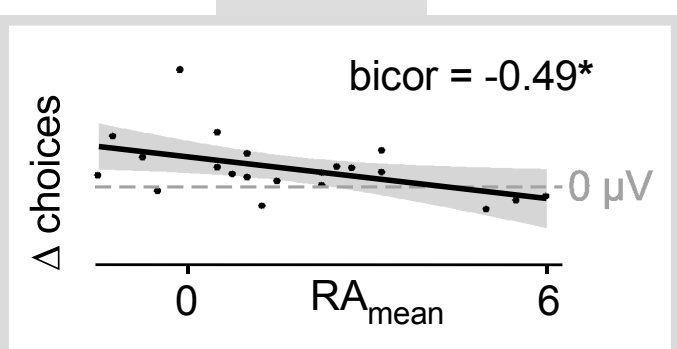

Figure 7: A. Grand average ERP average separately for risky and safe choices for strictly risk averse (RA group in blue, $R A_{\text {mean }}>2$ and $R A_{\text {low } E V}>0$ and, $R A_{\text {high } E V}>0, N=9$ ) and risk neutral participants ( $R N$ group, abs $\left.\left(R A_{\text {mean }}\right)<1, N=9\right)$ at the right parieto-central cluster. $B$. On the $y$-axis: difference between the late parietal component mean amplitude during risky - safe decision at the right cluster of electrode sites. On the $x$-axis: $R A_{\text {mean }}$. Biweight midcorrelation coefficient (bicor) is specified. ${ }^{*} p<.05$.

\section{Discussion}

We investigated the temporally resolved neural signatures of risky decision making, risk attitude and choice consistency. As expected, most of our participants were, on average, averse to risk. Importantly, measures of choice consistency and risk aversion between contexts captured behavioral heterogeneity in our sample. There was some super-imposition between choice consistency and risk aversion, with most risk-averse individuals having low level of consistency. Risk-neutral individuals included some individuals with low consistency, but the vast majority of this group was made up of individuals with high levels 
of choice consistency across trials. This is consistent with the idea that risk-aversion may be exhibited by individuals whose approach to decision-making is based on relatively fuzzy heuristics, whereas the adoption of more automatized heuristics may lead to a less riskaverse behavior.

This heterogeneity in behavior was reflected in marked differences in the ERP activity in response to the presentation of the options for a particular trial. In general, participants with greater choice consistency exhibited an enhanced frontal P200 component relative to those with less consistent choices. In contrast, individuals who were more risk-averse showed enhanced fronto-central MFN and increased centro-parietal P300/LPP. Note that then N1 component did not differ markedly in relation with the behavior pattern of individuals, suggesting that the difference in behavior are not due to differences in the attention posed to the stimuli. Summarizing, our results suggest that both risk aversion and choice consistency are suitable dimensions to study individual differences in the brain dynamics associated with risky decision making.

\section{Expected value maximization and the maintenance of internal}

\section{representations}

To explain individual differences in decision making, the values giving rise to choices are thought to be subjective. A rational individual will consistently maximize subjective value, but not necessarily the objective value expected from each possible choice's outcomes. If the internal goal is expected value maximization, individuals would be risk neutral and highly consistent. In our study, both consistency and risk neutrality were associated with a larger 
frontal P200. Our results also suggest smaller P300/LPP for individuals with low risk aversion, although that result is weaker as it is not robust to the exclusion of risk-seeking participants. This neural pattern can be interpreted in the context of developing automaticity in building and maintaining internal representations. In fact, this same pattern (increasing frontal P200 and decreasing P300/LPP) has been associated with the context updating of representations (Lenartowicz, Escobedo-Quiroz, \& Cohen, 2010). Moreover, the P300/LPP component is thought to reflect a resource-limited neural mechanism involved in the maintenance and updating of representations in working memory (Brumback, Low, Gratton, \& Fabiani, 2006; Peltz, Gratton, \& Fabiani, 2011). Consistently, individuals with absolute pitch process tones without producing a P300/LPP (Klein, 1984). They are proposed to have a set of internal "standards" that allow them to process tones without comparing a new representation with a previously heard benchmark representation (Klein, Coles, \& Donchin, 1984). Relatedly, the guided activation theory of the PFC (Miller \& Cohen, 2001) proposes that the PFC helps to maintain patterns of activity that represent goals and the means to achieve them. Thus, maintaining representations of value and risk and treating expected value as internal standard may facilitate consistency and value maximization.

For individuals who maintain stable value and goal representations throughout a decision task, one would expect relatively high choice consistency. Our correlation results suggest that this expectation holds at least for individuals either maintaining stable internal representations of the expected value over time, or generating such representations in a rapid, automatized fashion. By extension, consistent individuals may maintain (or quickly create) clearer representations of the subjective value of the risky choice option throughout the task. This ability implies that they have a reduced need to employ resource-limited and 
computationally-expensive processes to update internal representations, which would be indexed by the P300/LPP component. Also, the lack of uncertainty left by the more reliable representations in these individuals means that they may be better at emotion regulation later on (Myruski, Bonanno, Cho, Fan, \& Dennis-Tiwary, 2019). A comparable late effect is proposed to reflect a top-down modulation by the DLPFC of ventromedial PFC regions during decision making requiring self-control (Harris, Hare, \& Rangel, 2013). Further research should combine high temporal with high spatial resolution to precisely pinpoint the neural activities that enable stable representations during risky decision making.

\section{Medial frontal activity reflects risk perception}

We found larger MFN activity and less pronounced risk levels dissociation at low EV with increasing risk aversion. These results are in line with the reported overestimation of prospective risk by risk averters, that was found to correlate with a stronger response in the ventral striatum and in the anterior insula. Such an overestimation goes along with a difficulty to dissociate between gambles that are more or less risky than expected in the anterior insula, the inferior frontal gyrus and the ACC (Rudorf, Preuschoff, \& Weber, 2012). The MFN has been associated with the interaction between the PFC and the ACC in action monitoring (Gehring \& Knight, a, 2000). Botvinick and colleagues (Botvinick, Nystrom, Fissell, Carter, \& Cohen, 1999) proposed a role for the ACC in detecting and signaling conflicts for the recruitment of attention, and, in turn, of cognitive control. As could be expected, response times in our sample were longest when the subjective value of the two options was most similar, i.e., when the outcome of the safe option was close to the certainty equivalent. Response time is thought to reflect the level of conflict between two alternative choice 
options (Yamagishi, et al., 2017). Accordingly, our risky decision-making task could be considered as a demanding situation that requires conflict monitoring and action selection. An alternative interpretation of the MFN is that it reflects the emotional assessment (Luu, Collins, \& Tucker, 2000; Gehring \& Willoughby, The medial frontal cortex and the rapid processing of monetary gains and losses, 2002) and evaluation of (prospective) outcomes along a good-bad dimension (Nieuwenhuis, Yeung, Holroyd, Schurger, \& Cohen, 2004). It is possible that risk compared to risk neutral individuals, risk averse individuals assign risky options with greater emotional relevance, which could contribute to the modulation of the late positive potential by risk aversion in parietal sites.

\section{Decision-dependent signals in parietal electrodes}

Right posterior recording sites showed a dissociation between safe and risky choices. For the P300/LPP component, this dissociation depended on mean risk aversion, such that the relative increase in activity before a risky compared to a safe choice decreased as risk aversion increased. There is previous evidence for a fronto-parietal network with right dominance that plays a role in the control of visual attention (Corbetta \& Shulman, 2002). One could speculate that a shift of visual attention towards the risky option facilitates choice of the risky option in more risk tolerant individuals.

Exploratory analyses (Supplementary material) revealed a negative relation between within-condition consistency and the difference in risk aversion between the two EV conditions for two thirds of the sample. The rest of the sample (i.e., inconsistent group 2) did not show such a relation. This difference was accompanied by a weaker early posterior N1 component. Based on the strong evidence for a coupling between visual attention and 
saccade control (Deubel \& Schneider, 1996), it is possible that individuals in the inconsistent group 2 differ in early visual attention mechanisms from the other two groups. Further research with larger samples is needed to clarify this possibility.

\section{Limitations}

Kahneman and Tversky (Kahneman \& Tversky, 1979) proposes that when individuals evaluate a choice situation, they do not compare the absolute values of outcomes but the relative gain or losses with regard to a reference point (the outcome you feel entitled to). In our study, the value of the safe option could be considered as the reference point of the choice. When we manipulate the range of outcomes for risky options with constant expected value, the range of relative gains and losses will vary. If we consider a safe value of 50 and the two risky alternatives $(40,80)$ and $(30,90)$, the possible gain (respectively possible loss) would be 30 , i.e. $80-50$, (respectively $50-40=10$ for the loss) in the low risk level condition but 40 (respectively 20 for the loss) in the high risk level condition. In that example, the gain-to-loss ratio is higher in the low-risk condition than in the high-risk condition. Following (Tversky \& Kahneman, 1992), the gain-to-loss ratio is associated with loss aversion which has a strong impact on behavior. Therefore, it is possible that conditions with higher gain-to-loss ratio can have a stronger effect on choice behavior. This might explain why we find a significant effect of EV difference on the P200 in the low risk condition but not in the high risk condition. Further studies may want to separate the effects of risk levels, magnitude and gain-to-loss ratio. 
The safe option outcomes were distributed according to a pseudo-normal distribution centered on the average certainty equivalents reported by Holper and colleagues (Holper, et al., 2017). This manipulation ensures a similar number of risky and safe choices on average and presentation of the same safe options for all participants. However, by design, it imposes that risk averse individuals choose the safe options more often than risk tolerant individuals. The comparison between risky and safe choices may suffer from this imbalance. One possibility to address the issue is to tailor the safe options to each individual certainty equivalent (Christopoulos, Tobler, Bossaerts, Dolan, \& Schultz, 2009), but this has the disadvantage of using different safe options for different participants, which makes it hard to compare option-induced responses.

This study was not designed to explicitly investigate the effects of age and gender, although their influence was taken into account in our robust regression analyses. Both variables impacted the frontal P200 and the medial frontal negativity component. The effect of age on P200 is consistent with the literature (Bourisly \& Shuaib, 2018). There is yet no consensus on the effects of gender on MFN amplitude (Sandre, Banica, Riesel, Flake, Klawohn, \& Weinberg, 2020). A valuable extension of our study would be to assess whether the reported gender differences in risk aversion (Borghans, Heckman, Golsteyn, \& Meijers, 2009) replicate in a larger sample using our task and relate them to medial frontal structures. Relatedly, a further limitation of our study is that EEG has limited spatial specificity. It is therefore difficult to relate our results to fMRI reports relating IPFC activity to risk attitude. Based on our results, we expect the frontal P200 to be a good candidate for a neural marker of these effects. Research combining EEG with fMRI may shed more light on the issue. 
bioRxiv preprint doi: https://doi.org/10.1101/2021.05.10.443362; this version posted May $10,2021$. The copyright holder for this preprint

(which was not certified by peer review) is the author/funder, who has granted bioRxiv a license to display the preprint in perpetuity. It is made available under aCC-BY-NC-ND 4.0 International license.

Finally, the sample size in the current is relatively small, in particular for an individual difference study. For this reason, some of the conclusions of this study should be considered as preliminary, leaving more definite conclusion to future, more strongly powered studies.

\section{Conclusion}

Choice consistency is the hallmark of rationality. In our risky decision-making task, we find that stronger consistency correlates with an early expected value signal associated with cognitive control. Moreover, the MFN was associated with the subjective perception of risk, where overestimation could lead to risk avoidant behavior. Overall, this study identifies timeresolved components relevant for risky decisions and suggests that individual differences in value signals relate to individual differences in consistency. 


\section{References}

Bargh, J. A., \& Chartrand, T. L. (1999). The unbearable automaticity of being. American psychologist, 54 (7), 462.

Bartra, O., McGuire, J. T., \& Kable, J. W. (2013). The valuation system: a coordinate-based meta-analysis of BOLD fMRI experiments examining neural correlates of subjective value. Neuroimage , 76, 412-427.

Blavatskyy, P. R., \& Pogrebna, G. (2010). Models of stochastic choice and decision theories: Why both are important for analyzing decisions. Journal of Applied Econometrics , 25 (6), 963-986.

Borghans, L., Heckman, J. J., Golsteyn, B. H., \& Meijers, H. (2009). Gender Differences in Risk Aversion and Ambiguity Aversion. Journal of the European Economic Association , 7 (2-3), 649-658.

Bossaerts, P. (2010). Risk and risk prediction error signals in anterior insula. Brain structure \& function , 214 (5-6), 645-653.

Botvinick, M. M., Braver, T. S., Barch, D. M., Carter, C. S., \& Cohen, J. D. (2001). Conflict monitoring and cognitive control. Psychological Review , 108 (3), 624.

Botvinick, M., Nystrom, L. E., Fissell, K., Carter, C. S., \& Cohen, J. D. (1999). Conflict monitoring versus selection-for-action in anterior cingulate cortex. Nature , 402 (6758), 179-181.

Bourisly, A. K., \& Shuaib, A. (2018). Neurophysiological Effects of Aging: A P200 ERP Study. Translational neuroscience, 61-66.

Brumback, C. R., Low, K. A., Gratton, G., \& Fabiani, M. (2006). Putting Things into Perspective . Experimental Psychology, 52 (1), 21-30.

Buysse, D. J., Reynolds 3rd, C. F., Monk, T. H., Berman, S. R., \& Kupfer, D. J. (1989). The Pittsburgh Sleep Quality Index: a new instrument for psychiatric practice and research. Psychiatry Res , 28 (2), 193-213.

Chandrakumar, D., Feuerriegel, D., Bode, S., Grech, M., \& Keage, H. A. (2018). Event-related potentials in relation to risk-taking: A systematic review. Frontiers in Behavioral Neuroscience, 12, 111.

Christopoulos, G. I., Tobler, P. N., Bossaerts, P., Dolan, R. J., \& Schultz, W. (2009). Neural correlates of value, risk, and risk aversion contributing to decision making under risk. Journal of Neuroscience , 29 (40), 12574-12583.

Coles, M. G., Gratton, G., Bashore, T. R., Eriksen, C. W., \& Donchin, E. (1985). A psychophysiological investigation of the continuous flow model of human information processing. J Exp Psychol Hum Percept Perform , 11 (5), 529-53.

Corbetta, M., \& Shulman, G. L. (2002). Control of goal-directed and stimulus-driven attention in the brain. Nature reviews. Neuroscience, 3 (3), 201-215.

Delorme, A., \& Makeig, S. (2004). EEGLAB: an open source toolbox for analysis of single-trial EEG dynamics including independent component analysis. J Neurosci Methods , 134 (1), 921. 
Deubel, H., \& Schneider, W. X. (1996). Saccade target selection and object recognition: evidence for a common attentional mechanism. Vision research , 36 (12), 1827-1837. Donchin, E., \& Coles, M. G. (1988). Is the P300 component a manifestation of context updating. Behavioral and brain sciences , 11 (3), 357-427.

Dorris, M. C., \& Glimcher, P. W. (2004). Activity in posterior parietal cortex is correlated with the relative subjective desirability of action. Neuron, 44 (2), 365-78.

Eimer, M. (1998). Mechanisms of visuospatial attention: Evidence from event-related brain potentials. Visual Cognition , 5 (1-2), 257-286.

Emmorey, K., Midgley, K. J., Kohen, C. B., Sehyr, Z. S., \& Holcomb, P. J. (2017). The N170 ERP component differs in laterality, distribution, and association with continuous reading measures for deaf and hearing readers. Neuropsychologia , 106, 298-309.

Fecteau, S., Knoch, D., Fregni, F., Sultani, N., Boggio, P., \& Pascual-Leone, A. (2007).

Diminishing risk-taking behavior by modulating activity in the prefrontal cortex: A direct current stimulation study. Journal of Neuroscience, 27 (46), 12500-12505.

Fernandes, C., Pasion, R., Gonçalves, A. R., Ferreira-Santos, F., Barbosa, F., Martins, I. P., et al. (2018). Age differences in neural correlates of feedback processing after economic decisions under risk. Neurobiology of Aging , 65, 51-59.

Frey, D., Johnson, E. D., \& De Neys, W. (2018). Individual differences in conflict detection during reasoning. Quarterly journal of experimental psychology , 71 (5), 1188-1208.

Gehring, W. J., \& Knight, R. T. (2000). Prefrontal-cingulate interactions in action monitoring. Nature neuroscience, 3 (5), 516-520.

Gehring, W. J., \& Willoughby, A. R. (2002). The medial frontal cortex and the rapid processing of monetary gains and losses. Science , 295 (5563), 2279-2282.

Gehring, W. J., \& Willoughby, A. R. (2002). The medial frontal cortex and the rapid processing of monetary gains and losses. Science, 295 (5563), 2279-2282.

Gratton, G., Coles, M. G., \& Donchin, E. (1983). A new method for off-line removal of ocular artifact. Electroencephalogr Clin Neurophysiol , 55 (4), 468-484.

Grether, D. M., \& Plott, C. R. (1979). Economic theory of choice and the preference reversal phenomenon. American Economic Review , 69 (4), 623-638.

Gu, R., Zhang, D., Luo, Y. a., \& Broster, L. S. (2018). Predicting risk decisions in a modified Balloon Analogue Risk Task: Conventional and single-trial ERP analyses. Cognitive, affective \& behavioral neuroscience, 18 (1), 99-116.

Hajcak, G., Dunning, J. P., \& Foti, D. (2009). Motivated and controlled attention to emotion: time-course of the late positive potential. Clinical neurophysiology, 120 (3), 505-510.

Harless, D. W., \& Camerer, C. F. (1994). The Predictive Utility of Generalized Expected Utility Theories. Econometrica , 1251-1289.

Harris, A., Hare, T., \& Rangel, A. (2013). Temporally dissociable mechanisms of self-control: early attentional filtering versus late value modulation. The Journal of neuroscience , 33 (48), 18917-31.

Hey, J. D. (2001). Does repetition improve consistency? Experimental Economics , 4 (1), 5 54. 
Hey, J. D., \& Orme, C. (1994). Investigating generalizations of expected utility theory using experimental data. Econometrica: Journal of the Econometric Society , 1291-1326.

Hillyard, S. A., Teder-Sälejärvi, W. A., \& Münte, T. F. (1998). Temporal dynamics of early perceptual processing. Current opinion in neurobiology , 8 (2), 202-210.

Holper, L., Van Brussel, L. D., Schmidt, L., Schulthess, S., Burke, C. J., Louie, K., et al. (2017). Adaptive Value Normalization in the Prefrontal Cortex Is Reduced by Memory Load. eNeuro , 4 (2), 1.

Holper, L., Wolf, M., \& Tobler, P. N. (2014). Comparison of functional near-infrared spectroscopy and electrodermal activity in assessing objective versus subjective risk during risky financial decisions. Neuroimage , 84, 833-842.

Intriligator, M. D. (1973). A probabilistic model of social choice. Review of Economic Studies, 40 (4), 553-560.

Kable, J. W., \& Glimcher, P. W. (2009). The neurobiology of decision: consensus and controversy. Neuron, 63 (6), 733-45.

Kachelmeier, S. J., \& Shehata, M. (1992). Examining Risk Preferences Under High Monetary Incentives : Experimental Evidence from the People's Republic of China. American Economic Review , 1120-1141.

Kahneman, D., \& Tversky, A. (1979). Prospect Theory: An Analysis of Decision under Risk. Econometrica , 47 (2), 263-292.

Klein, M., Coles, M. G., \& Donchin, E. (1984). People with absolute pitch process tones without producing a p300. Science, 223 (4642), 1306-9.

Knoch, D., Gianotti, L. R., Pascual-Leone, A., Treyer, V., Regard, M., Hohmann, M., et al. (2006). Disruption of right prefrontal cortex by low-frequency repetitive transcranial magnetic stimulation induces risk-taking behavior. Journal of Neuroscience , 26 (24), 64696472.

Kroenke, K., Spitzer, R. L., \& Williams, J. B. (2001). The PHQ-9: validity of a brief depression severity measure. Journal of general internal medicine , 16 (9), 606-613.

Kurtz-David, V., Persitz, D., Webb, R., \& Levy, D. J. (2019). The neural computation of inconsistent choice behavior. Nature Communications , 10 (1), 1-14.

Langfelder, P., \& Horvath, S. (2008, Dec). WGCNA: an R package for weighted correlation network analysis. BMC Bioinformatics , 559.

Lenartowicz, A., Escobedo-Quiroz, R., \& Cohen, J. D. (2010). Updating of context in working memory: an event-related potential study. Cognitive, affective \& behavioral neuroscience , 10 (2), 298-315.

Levy, I., Snell, J., Nelson, A. J., Rustichini, A., \& Glimcher, P. W. (2010). Neural representation of subjective value under risk and ambiguity. Journal of neurophysiology, 103 (2), 1036-47. Logan, G. D. (1988). Toward an instance theory of automatization. Psychological review , 95 (4), 492.

Long, J. A. (2021, April 7). jtools. Retrieved from RDocumentation:

https://rdocumentation.org/packages/jtools/versions/2.1.3

Lopez-Calderon, J., \& Luck, S. J. (2014). ERPLAB: an open-source toolbox for the analysis of event-related potentials. Frontiers in human neuroscience , 8, 213. 
Louie, K., Glimcher, P. W., \& Webb, R. (2015). Adaptive neural coding: from biological to behavioral decision-making. Current opinion in behavioral sciences , 5, 91-99.

Louie, K., Grattan, L. E., \& Glimcher, P. W. (2011). Reward value-based gain control: divisive normalization in parietal cortex. The Journal of neuroscience, 31 (29), 10627-39.

Louie, K., Khaw, M. W., \& Glimcher, P. W. (2013). Normalization is a general neural mechanism for context-dependent decision making. Proceedings of the National Academy of Sciences of the United States of America , 110 (15), 6139-44.

Luce, R. D. (1999). Utility of Gains and Losses, Ch. 1. New York: Psychology Press.

Luck, S. J., \& Hillyard, S. A. (1994). Electrophysiological correlates of feature analysis during visual search. Psychophysiology , 31 (3), 291-308.

Luck, S. J., Stewart, A. X., Simmons, A. M., \& Rhemtulla, M. (2021, Mar). Standardized measurement error: A universal metric of data quality for averaged event-related potentials. Psychophysiology, Psychophysiology.

Luu, P., Collins, P., \& Tucker, D. M. (2000). Mood, personality, and self-monitoring: negative affect and emotionality in relation to frontal lobe mechanisms of error monitoring. Journal of experimental psychology. General, 129 (1), 43-60.

Mair, P., \& Wilcox, R. (2019). Robust statistical methods in R using the WRS2 package. Behavior research methods , 1-25.

Mattsson, L. G., \& Weibull, J. W. (2002). Probabilistic choice and procedurally bounded rationality. (1, Ed.) Games and Economic Behavior , 41, 61-78.

McCoy, A. N., \& Platt, M. L. (2005). Risk-sensitive neurons in macaque posterior cingulate cortex. Nature neuroscience , 8 (9), 1220-7.

McFadden, D. L. (2005). Revealed stochastic preference: a synthesis. Economic Theory , 26 (2), 245-264.

Miller, E. K., \& Cohen, J. D. (2001). An integrative theory of prefrontal cortex function. Annual Review of Neuroscience, 24, 167-202.

Myruski, S., Bonanno, G. A., Cho, H., Fan, B., \& Dennis-Tiwary, T. A. (2019). The late positive potential as a neurocognitive index of emotion regulatory flexibility. Biological psychology, $148,107768$.

Nieuwenhuis, S., Aston-Jones, G., \& Cohen, J. D. (2005). Decision making, the P3, and the locus coeruleus-norepinephrine system. Psychological bulletin , 131 (4), 510.

Nieuwenhuis, S., Schweizer, T. S., Mars, R. B., Botvinick, M. M., \& Hajcak, G. (2008). A framework for studying the neurobiology of value-based decision making. Nature Reviews Neuroscience , 9 (7), 545-556.

Nieuwenhuis, S., Yeung, N., Holroyd, C. B., Schurger, A., \& Cohen, J. D. (2004). Sensitivity of electrophysiological activity from medial frontal cortex to utilitarian and performance feedback. Cerebral Cortex , 14 (7), 741-747.

Peltz, C. B., Gratton, G., \& Fabiani, M. (2011). Age-related changes in electrophysiological and neuropsychological indices of working memory, attention control, and cognitive flexibility. Frontiers in Psychology , 2, 190.

Platt, M. L., \& Glimcher, P. W. (1999). Neural correlates of decision variables in parietal cortex. Nature , 400 (6741), 233-238. 
Polezzi, D., Sartori, G., Rumiati, R., Vidotto, G., \& Daum, I. (2010). Brain correlates of risky decision-making. Neuroimage , 49 (2), 1886-94.

Rangel, A., Camerer, C., \& Montague, P. R. (2008). A framework for studying the neurobiology of value-based decision makin. Nature Reviews Neuroscience, 9 (7), 545-556. Rieskamp, J. (2008). The Probabilistic Nature of Preferential Choice. Journal of Experimental Psychology: Learning Memory and Cognition , 34 (6), 1446.

Rudorf, S., Preuschoff, K., \& Weber, B. (2012). Neural correlates of anticipation risk reflect risk preferences. The Journal of neuroscience , 32 (47), 16683-92.

Ryan, M. (2018). Uncertainty and binary stochastic choice. Economic Theory, 65 (3), 629662.

Sandre, A., Banica, I., Riesel, A., Flake, J., Klawohn, J., \& Weinberg, A. (2020). Comparing the effects of different methodological decisions on the error-related negativity and its association with behaviour and gender. International journal of psychophysiology , 156, 1839.

Schuermann, B., Endrass, T., \& Kathmann, N. (2012). Neural correlates of feedback processing in decision-making under risk. Frontiers in Human Neuroscience , 6, 204. Seidl, C. (2002). Preference reversal. Journal of Economic Surveys, 16 (5), 621-655. Spitzer, R. L., Kroenke, K., Williams, J. B., \& Löwe, B. (2006). A brief measure for assessing generalized anxiety disorder: the GAD-7. Archives of internal medicine , 166 (10), 1092-7.

Strasburger, H. (2001). Converting between measures of slope of the psychometric function. Perception \& psychophysics , 63, 1348-55.

Sugrue, L. P., Corrado, G. S., \& Newsome, W. T. (2004). Matching behavior and the representation of value in the parietal cortex. Science, 304 (5678), 1782-7.

Testa, G., Buongiorno, F., Rusconi, M. L., Mapelli, D., Vettor, R., Angeli, P., et al. (2020). ERP correlates of cognitive control and food-related processing in normal weight and severely obese candidates for bariatric surgery: Data gathered using a newly designed Simon task. Biological psychology, 149, 107804.

Tobler, P. N., Christopoulos, G. I., O'Doherty, J. P., Dolan, R. J., \& Schultz, W. (2009). Riskdependent reward value signal in human prefrontal cortex. Proc Natl Acad Sci U S A, 106 (17), 7185-7190.

Tobler, P. N., O'Doherty, J. P., Dolan, R. J., \& Schultz, W. (2007). Reward value coding distinct from risk attitude-related uncertainty coding in human reward systems. Journal of Neurophysiology, 97 (2), 1621-1632.

Tsetsos, K., Usher, M., \& Chater, N. (2010). Preference Reversal in Multiattribute Choice. Psychological Review , 117 (4), 1275.

Tversky, A., \& Kahneman, D. (1992). Prospect theory : Cumulative Representation of uncertainty. Journal of Risk and Certainty , 4 (5), 297-324.

Wang, G., Li, J., Wang, P., Zhu, C., Pan, J., \& Li, S. (2019). Neural dynamics of processing probability weight and monetary magnitude in the evaluation of a risky reward. Frontiers in Psychology , 10, 554. 
Webb, R., Levy, I., Lazzaro, S. C., Rutledge, R. B., \& Glimcher, P. W. (2019). Neural random utility: Relating cardinal neural observables to stochastic choice behavior. Journal of Neuroscience, Psychology, and Economics , 12 (1), 45.

Xu, S., Pan, Y., Wang, Y., Spaeth, A. M., Qu, Z., \& Rao, H. (2016). Real and hypothetical monetary rewards modulate risk taking in the brain. Scientific Reports , 6, 29520.

Yamagishi, T., Matsumoto, Y., Kiyonari, T., Takagishi, H., Li, Y., Kanai, R., et al. (2017). Response time in economic games reflects different types of decision conflict for prosocial and proself individuals. Proceedings of the National Academy of Sciences of the United States of America , 114 (24), 6394-6399.

Zhang, D., Gu, R., Broster, L. S., Jiang, Y., Luo, W., Zhang, J., et al. (2014). Linking brain electrical signals elicited by current outcomes with future risk decision-making. Front Behav Neurosci , 8, 84.

Zheng, Y., An, T., Li, Q., \& Xu, J. (2020). Distinct electrophysiological correlates between expected reward and risk processing. Psychophysiology , e13638.

Zheng, Y., Li, Q., Wang, K., Wu, H., \& Liu, X. (2015). Contextual valence modulates the neural dynamics of risk processing. Psychophysiology , 52 (7), 895-904.

Zilker, V., Hertwig, R., \& Pachur, T. (2020). Age differences in risk attitude are shaped by option complexity. Journal of experimental psychology. General , 149 (9), 1644-1683. 


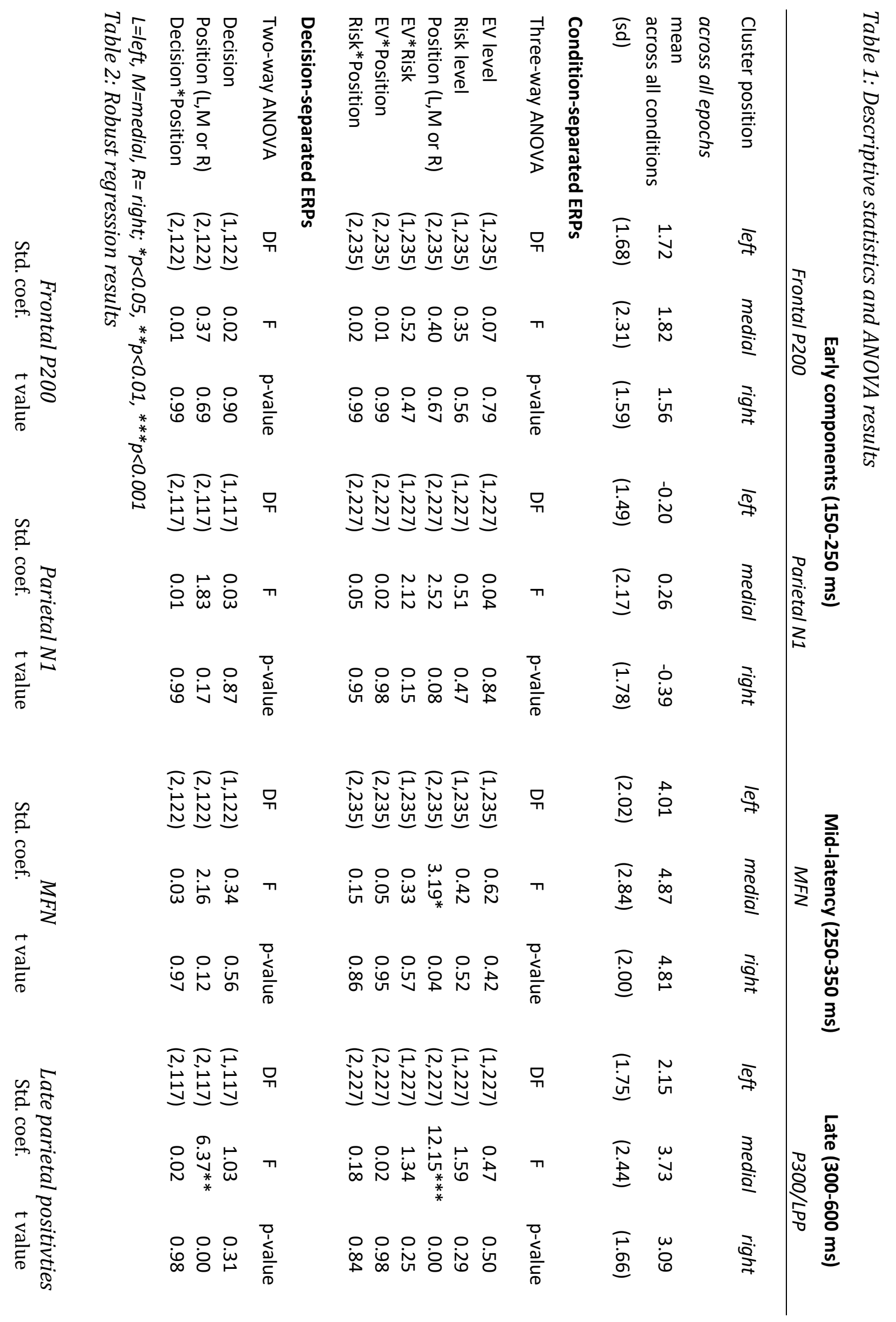




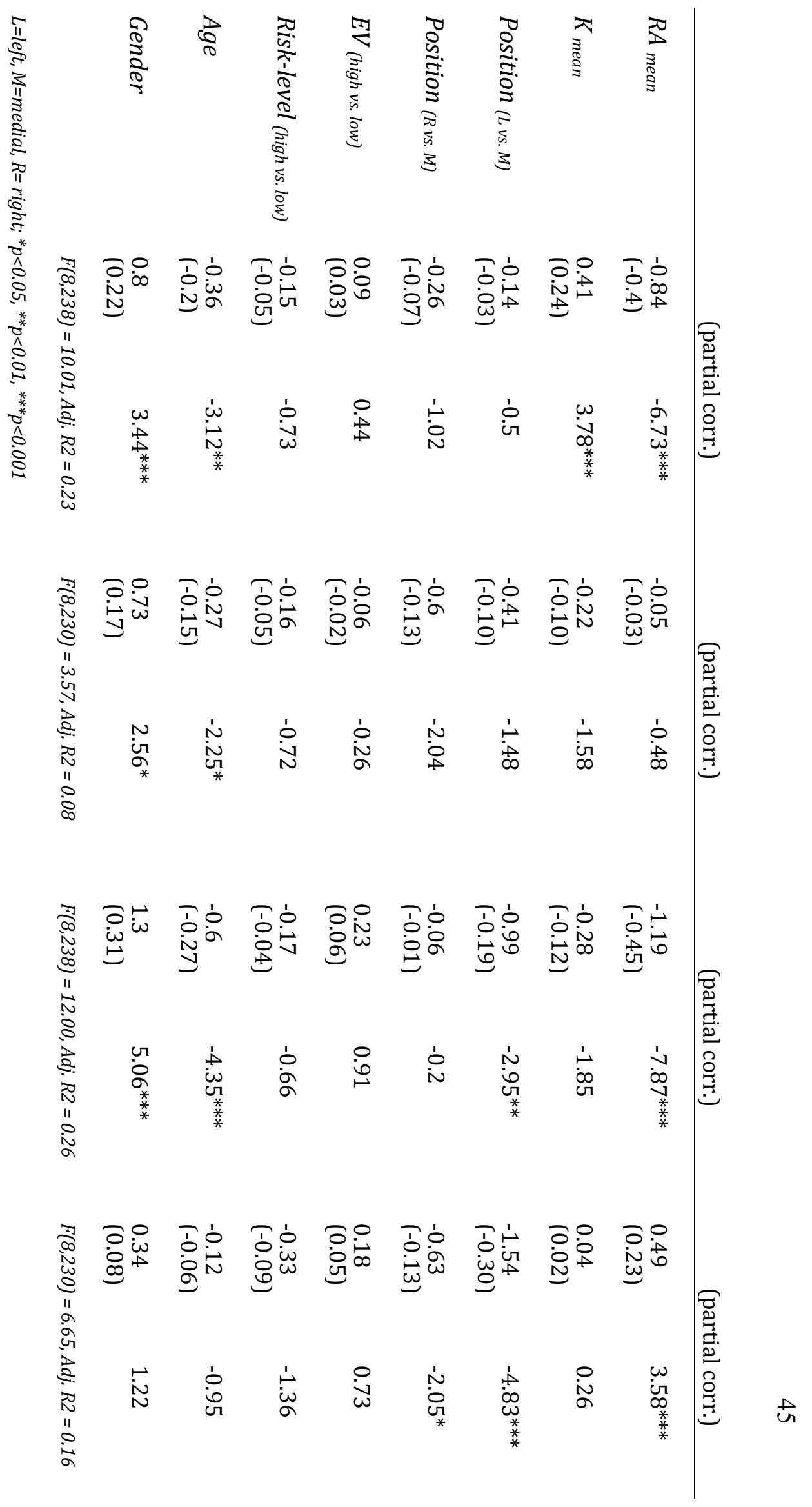


Table 3: biweight midcorrelation and slope estimates from robust linear regression between behavioral measurements and differences in signal between conditions at the medial frontocentral cluster

\begin{tabular}{|c|c|c|c|c|c|c|c|}
\hline & & \multicolumn{4}{|c|}{ P200 } & \multirow{2}{*}{\multicolumn{2}{|c|}{$\begin{array}{c}\text { MFN } \\
\text { RA mean }\end{array}$}} \\
\hline & & \multicolumn{2}{|c|}{ RA mean } & \multicolumn{2}{|c|}{ K mean } & & \\
\hline & & $\begin{array}{l}\text { bicor } \\
\text { slope }\end{array}$ & $\begin{array}{c}\mathrm{p} \\
\text { t value }\end{array}$ & $\begin{array}{l}\text { bicor } \\
\text { slope }\end{array}$ & $\begin{array}{c}\mathrm{p} \\
\text { t value }\end{array}$ & $\begin{array}{l}\text { bicor } \\
\text { slope }\end{array}$ & $\begin{array}{c}\mathrm{p} \\
\text { t value }\end{array}$ \\
\hline \multirow[t]{4}{*}{$\Delta$ Risk-level } & low EV & 0.39 & 0.07 & 0.05 & 0.81 & $0.44^{*}$ & 0.04 \\
\hline & & 0.37 & 2.03 & 0.01 & 0.29 & $0.39 *$ & 2.23 \\
\hline & high EV & 0.08 & 0.75 & -0.36 & 0.11 & 0.17 & 0.47 \\
\hline & & 0.08 & 0.66 & -0.02 & -1.61 & 0.11 & 0.64 \\
\hline \multirow[t]{4}{*}{$\Delta \mathbf{E V}$} & low risk & 0.21 & 0.35 & $0.48^{*}$ & 0.03 & 0.02 & 0.91 \\
\hline & & 0.15 & 0.94 & $0.02^{*}$ & 2.64 & 0.02 & 0.08 \\
\hline & high risk & -0.28 & 0.2 & -0.26 & 0.25 & -0.25 & 0.26 \\
\hline & & -0.10 & -0.69 & -0.01 & -1.50 & -0.16 & -0.96 \\
\hline
\end{tabular}

